\title{
Anchor Nodes Placement for Effective Passive Localization
}

\author{
Robert Akl, Karthik Pasupathy \\ Department of Computer Science and Engineering \\ University of North Texas \\ Denton, Texas, USA \\ Robert.Ak1@unt.edu, Karthikeyanp@unt.edu
}

\author{
Mohamad Haidar \\ Department of Electrical Engineering \\ Ecole de Technologie Superieure \\ Canada \\ mhaidar@hotmail.com
}

\begin{abstract}
In many applications, the exact location of the sensor nodes is unknown after deployment. Localization is a process used to locate sensor nodes' positional coordinates, which is vital information. The localization is generally assisted by anchor nodes that are also sensor nodes but with known locations. Anchor nodes generally are expensive and need to be optimally placed for effective localization. Passive localization is one of the localization techniques where the sensor nodes silently listen to the global events like thunder sounds, seismic waves, lighting, etc. According to previous studies, the ideal location to place anchor nodes was on the perimeter of the sensor network. This may not be the case in passive localization, since the function of anchor nodes here is different than the anchor nodes used in other localization systems. We do extensive studies on positioning anchor nodes for effective localization. Several simulations are run in dense and sparse networks for proper positioning of anchor nodes. We show that, for effective passive localization, the optimal placement of the anchor nodes is at the center of the network in such a way that no three anchor nodes share linearity. The more the non-linearity, the better the localization. The localization for our network design proves better when we place anchor nodes to form right angles.
\end{abstract}

Keywords- anchor nodes; wireless sensors; localization;

\section{INTRODUCTION}

A Wireless Sensor Network (WSN) is made of spatially distributed sensor nodes which are normally tiny in size and can be used to monitor environmental or physical conditions like pressure, temperature, audio, video, motion, etc. These sensor nodes typically have a low-power processor, a sensor board, a memory, a transceiver, and a power source of size similar to AA batteries [1].

WSNs generally consist of a base station, which can communicate wirelessly with the sensor nodes. These sensor nodes collect data, compress it, and transmit it to the base station directly or indirectly through other sensor nodes present in the network using routing algorithms [2].

The nodes may either store and transmit raw data to its base station or process and transmit processed data to its base station. As one can fathom, in the latter case, the node consumes more power, but it may be used in situations to avoid transmission time (hence more battery life). The nodes may transmit the raw data in applications where continuous data processing is involved. In mobile networks involving continuous sensing and positional tracking, the data is processed at the sensor node. In such networks, the sensor node cannot afford to wait until the base station processes and transmits the information back.

There are several localization techniques like Received Signal Strength, Time of Arrival, and Angle of Arrival. More details on these algorithms will be covered in the next section. Every technique has its own advantages and disadvantages depending on the application of WSN.

In our research, we have used passive localization, which uses the time differences between global events to localize the sensor nodes [3]. The main advantage of using passive localization is, as the name implies, the nodes have to just listen or measure the signal strength for localization. The signals could be global events occurring around the sensor bed like thunder, wind, tremor, etc. This localization technique like other techniques also requires anchor nodes. Anchor nodes are sensor nodes with known locations. This can be achieved by either equipping them with GPS or by carefully deploying them at known locations. Although passive localization uses anchor nodes, its function is slightly different from other localization techniques. Unlike anchor nodes in other techniques, anchor nodes in passive localization do not transmit signals for localization. They just listen for global events for localization. It is well known that increasing the number of anchor nodes gives rise to better localization; however, it may not be a feasible solution due to the extra hardware requirements that may be more expensive.

Some researchers have said that it is better to place anchors uniformly on the perimeter of the network [4], [5]. Nonetheless, it may not be the case in passive localization, since the signals are transmitted from global events that may be far away from the senor bed. Moreover, placing anchors with known locations and sensor nodes with unknown locations on the same perimeter makes less sense. It would be easier and less expensive to deploy anchor nodes within the network. We have run several simulations to study optimal placement of anchor nodes for effective localization. The purpose of optimal positions of anchor nodes is: (1) to reduce localization error, (2) to avoid excessive deployment costs. Deployment cost of anchor nodes needs to be considered since we need to know its geographical coordinates.

This paper is structured as follows: section II discusses briefly some of the common localization techniques. These techniques are then compared with each other. In section III, 
the details of passive localization and the importance of positioning of anchor nodes for localization is presented. Different parameters and strategies to consider while deploying anchor nodes are also detailed. The results of various simulations carried on sparse as well as dense WSNs to study the effects of anchor nodes' position on localization errors are presented in section IV. Finally, we conclude our study on positioning anchor nodes for effective passive localization in section $\mathrm{V}$ while presenting possible future directions to extend our research.

\section{LOCALIZATION}

Many applications need location information of sensor nodes and these positions can be acquired by using localization algorithms. There are two main approaches in localization: distributed and centralized system [6].

In a centralized system, individual nodes transmit all the localization information to a central server, which then computes the location of each of its nodes. One advantage of this approach is that all information is given to the central server. Therefore, the system is not limited by a specific algorithm to be used. This also gives rise to more accurate results, as the system may not have any limitation in processing the data. The main disadvantages of this system are traffic congestion and computational complexities.

On the other hand, in a distributed system, the localization is distributed across the network. Each node attempts to localize itself and helps in localizing other nodes. This process is usually iterative. The algorithms used are generally energy efficient and self-organizing.

The two main challenges for localization can be divided into network and channel parameters [6]. With respect to network parameters, the localization is affected by the size of nodes, anchors, topology, and network connectivity. Anchors are also nodes with known location. They may be either deployed at known locations or may be equipped with GPS. A network with dense nodes gives rise to better localization than a network with sparse nodes. This may be due to poorly connected nodes in sparse networks. It may be better to have dense nodes, but with increasing the number of nodes, the propagation error also increases.

In accordance to channel parameters, the localization techniques are also affected. Common localization techniques depend on Radio Frequency (RF) ranging. Some of the examples are Time of Arrival (TOA), Received Signal Strength (RSS), and Angle of Arrival (AOA). TOA techniques are more accurate than RSS techniques but the latter are more practical than TOA. The performance of these techniques varies when deployed in outdoor environments and indoor environments. This is due to the uncertainty of RF propagation, which may cause severe localization errors. To avoid these errors, the channel needs to be investigated for a compatible localization algorithm.

The localization system can be separated into three functional components [7]: distance and angle estimation, position computation, and localization algorithm.
The distance/angle estimation component estimates the distance or angle between two nodes. This information may be used by other components. The position computation component uses the distance or angle information of anchor nodes to estimate a node's position. The localization algorithm component is the heart of localization system. This component determines the manipulation of the available information to let other nodes estimate their position. Since every node in a sensor network is equipped with a radio for communication, this unit can be used for range estimation techniques.

\section{ANCHOR NODE PlACEMENT}

\section{A. Passive Localization}

Passive localization techniques use time differences of global events for localization. Some examples of such events are sound of thunder, seismic data, moving clouds, lightning, etc. Since the sensor nodes just sense the events and do not transmit while localizing, it is known as passive localization [3]. The time difference between each pair of nodes is proportional to the distances from the plane normal to the global event propagation and the sensor nodes. These time differences are known as projected distances.

The global events are first detected by looking for similar raw signals at all sensor nodes. The similarity is checked by measuring the correlation between them. The received signals between each node are shifted by a time interval due to propagation delay. The time difference of arrival from detected events is then calculated between each pair of nodes. These time shifts are nothing but the projected distances. The principal axes with largest variances are then computed from the set of projected distances. The position of the sensor nodes can then be obtained from linear combinations of the principal axes. The linear combination coefficients can be found using anchor nodes with known locations.

If we have $n$ nodes and $m$ sets of projected distances from each global event, then we can build a distance matrix $D$ as follows:

$$
\left[\begin{array}{ccccc}
u_{11} & u_{12} & \cdot & \cdot & u_{1 m} \\
u_{21} & \cdot & & & \cdot \\
\cdot & & \cdot & & \cdot \\
\cdot & & & \cdot & \cdot \\
u_{n 1} & \cdot & \cdot & \cdot & u_{n m}
\end{array}\right],
$$

where $u_{i j}=$ time at which signal arrives at node $i$ from event $j$.

This matrix can be decomposed to three other matrices using Singular Value Decomposition.

$$
D=P \cdot \Sigma \cdot Q
$$

The first two column vectors of matrix $P$ are the most important principal axes of $D$. Let these vectors be $\mathbf{p}_{\mathbf{1}}$ and $\mathbf{p}_{\mathbf{2}}$. Then the node's position $\left(x_{i}, y_{i}\right)$ can be obtained as follows:

$$
\begin{aligned}
& x_{i}=a_{\mathbf{1}} \cdot p_{\mathbf{1} i}+b_{\mathbf{1}} \cdot p_{\mathbf{2} i}+c_{\mathbf{1}}, \\
& y_{i}=a_{\mathbf{2}} \cdot p_{\mathbf{1} i}+b_{\mathbf{2}} \cdot p_{\mathbf{2} i}+c_{\mathbf{2}}
\end{aligned}
$$

where $\mathbf{p}_{\mathbf{i j}}$ is the $j^{\text {th }}$ element of vector $\mathbf{p}_{\mathbf{i}}, a_{1}, a_{2}, b_{1}$ and $b_{2}$ are the linear combination coefficients and $c_{1}$ and $c_{2}$ are the coefficient for the vector of ones. 
Having six unknown variables, we need at least three anchor nodes to obtain all the node positions. Using more than three anchor nodes can improve the redundancy in computing the linear combination coefficients [3].

To calculate the nodes' positions, let

$$
\begin{gathered}
\boldsymbol{s}=\left[\begin{array}{l}
a_{1} \\
b_{1} \\
c_{1}
\end{array}\right], \boldsymbol{t}=\left[\begin{array}{l}
a_{2} \\
b_{2} \\
c_{2}
\end{array}\right], \boldsymbol{x}=\left[\begin{array}{c}
x_{1} \\
\cdot \\
\cdot \\
\cdot \\
x_{k}
\end{array}\right], \boldsymbol{y}=\left[\begin{array}{c}
y_{1} \\
\cdot \\
\cdot \\
y_{k}
\end{array}\right], \\
A=\left[\begin{array}{ccc}
\mathbf{p}_{11} & \mathrm{p}_{2 \mathrm{k}} & 1 \\
\cdot & \cdot & \cdot \\
\cdot & \cdot & \cdot \\
\cdot & \cdot & \cdot \\
\mathbf{p}_{1 \mathrm{k}} & \mathrm{p}_{2 \mathrm{k}} & 1
\end{array}\right] \\
\boldsymbol{s}=A^{+} \cdot \boldsymbol{x}, \quad \boldsymbol{t}=A^{+} \cdot \boldsymbol{y},
\end{gathered}
$$

where $k$ is the number of anchor nodes and $A^{+}$is the pseudo inverse of matrix $A$ [8]. After computing the linear coefficients, we can obtain the position of each node by using (3).

\section{B. Anchor Node Placement}

Conventionally several researchers have observed that anchor nodes should be uniformly spread around the perimeter of the sensor network [5]. As the deployment of anchor nodes are at unknown location and due to other conditions, it may not be possible to deploy anchor nodes uniformly at the perimeter of the sensor network.

No matter how the network is set up, the error associated with localization is inevitable. There may be various sources for this error, one of which is the unavailability of anchor nodes. This may arise due to low deployment density or poor signal propagation due to factors like multipath effects, fading effects, dead zones, and poor visibility [1].

The propagation of radio waves is unpredictable in practice making it sensitive to errors. The errors may also rise due to erratic measurements depending on hardware quality, time synchronization accuracy, and ranging techniques used. The other source of error arises due to anchor node's position.

Several articles have been published on localization techniques and few articles address effective positioning of anchor nodes. This may be due to their focus on the localization techniques rather than parameters affecting localization.

In passive localization, unlike other localization techniques, the nodes are passive meaning they do not transmit and instead silently sense all the events happening around the network. Therefore, the function of the anchor nodes in passive localization is slightly different from other localization methods. We have examined the effect of anchor node's positions on passive localization. Anchor node's placement and geometry play a crucial role on localization.

\section{1) Density of the Anchor Nodes}

Increasing the density of the anchor nodes may minimize localization error, but the cost of deployment and hardware increases. The density of anchor nodes may still be increased to leverage redundancy.

2) Geometry of the Anchor Nodes

Geometry of the anchor nodes also affects localization. Deploying more than two anchor nodes in a line may not improve localization but may be used for fault tolerance.

We have conducted several simulations to study the positioning of anchor nodes for effective passive localization.

\section{SimUlation AND RESUltS}

\section{A. Assumptions}

For the sake of simplicity, the following assumptions were taken into consideration:

- The propagation velocity of all global signals is unity.

- The clocks within each node are time synchronized.

- Global events are randomly distributed over the network.

- Node failure was ignored to assume an ideal case. However, in the real world redundancy is considered to manage node failures.

- All the global event sources are static.

\section{B. Localization Error}

Every localization technique is associated with an error, which is inevitable. Localization error $\left(L_{e r r}\right)$ is the positioning error obtained from localization algorithms. In our simulations, we define $L_{\text {err }}$ as

$$
L_{e r r}=\left(1-\frac{1}{|N|} \sum_{i, j \in N} \frac{d_{i j}}{D_{i j}}\right) 100 \%
$$

where $N$ is the index set of all nodes, $d_{i j}$ and $D_{i j}$ are the measured and the actual distances between node $i$ and $j$.

\section{Random Deployment}

We ran several simulations to observe the dependence of anchors and events on passive localization. Results are presented in Fig. 1 and Fig. 2.

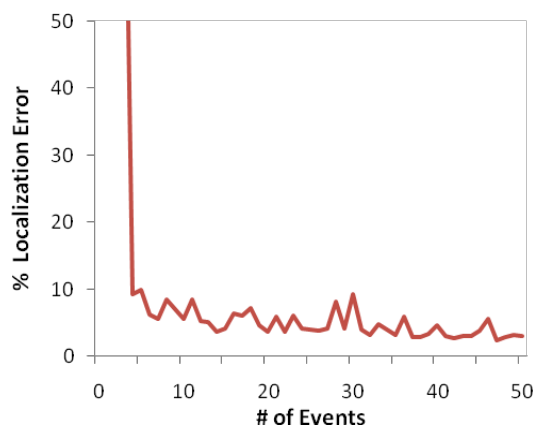

Fig. 1. Dependence of number of events on localization (deployment is random). 


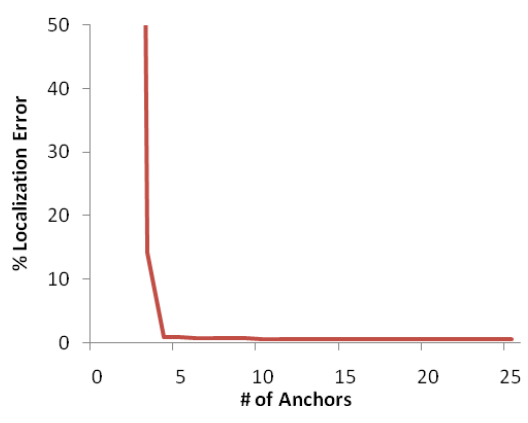

Fig. 2. Dependence of number of anchors on localization (deployment is nonrandom).

In these simulations, each result was obtained from an average of 3000 simulations. In each simulation, about 50 events and 25 nodes were randomly scattered in a network. To look at the dependence of number of events on localization, three anchor nodes were randomly chosen at each time. As can be seen in Fig. 1, the localization improves as we increase the number of events around the sensor network. One can also see that increasing the number of anchor nodes improves the localization, Fig. 2. Minimal localization improvement was achieved after 5-6 anchor nodes as the localization error approached zero. Moreover, using additional anchor nodes provides no further benefit unless redundancy or fault tolerance is considered.

The following section investigates the effect of sparse (less dense) networks on anchor node placement.

\section{Manual Deployment}

We simulated passive localization on a network of 25 nodes arranged in a $5 \times 5$ grid as shown in Fig. 3. The locations of the anchor nodes were manually fixed.

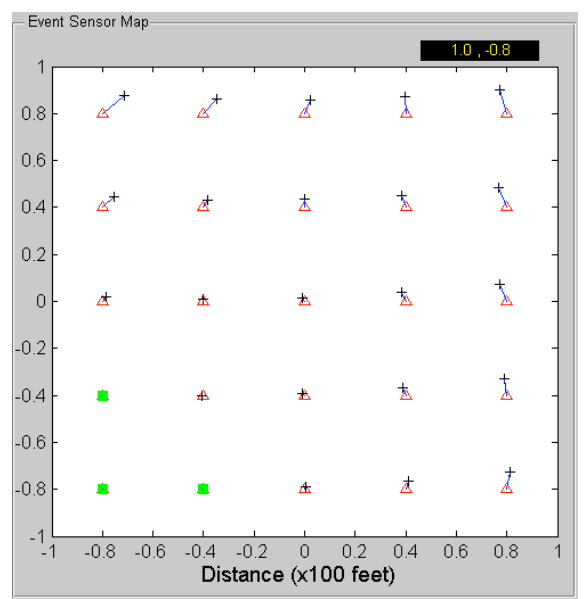

Fig. 3. Localization of a sparse network with anchor nodes (squares) at a corner of a sensor bed containing sensor nodes (triangles) and their estimated positions (crosses).

As can be seen, the sensor nodes that are far away from the anchor nodes are prone to localization error. The localization error $\left(L_{\text {err }}\right)$ is $15.52 \%$ for the above setup.

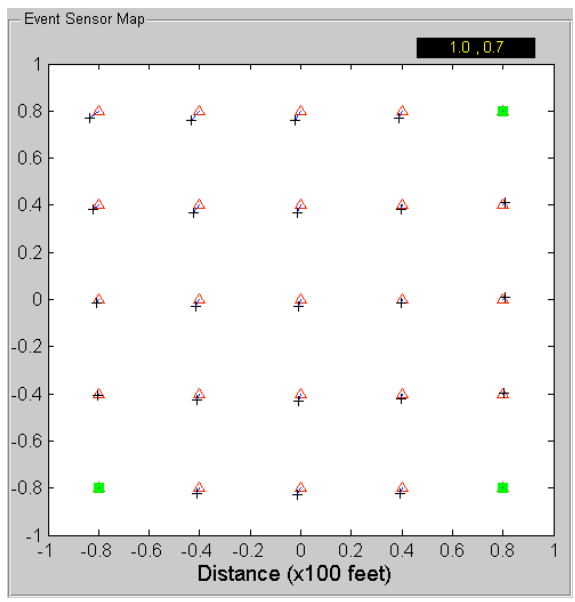

Fig. 4. Localization on a sparse network with anchor nodes deployed on the perimeter of the sensor bed.

A localization error of $9.68 \%$ was achieved in Fig. 4 . The localization error decreased when the anchors were placed on the perimeter.

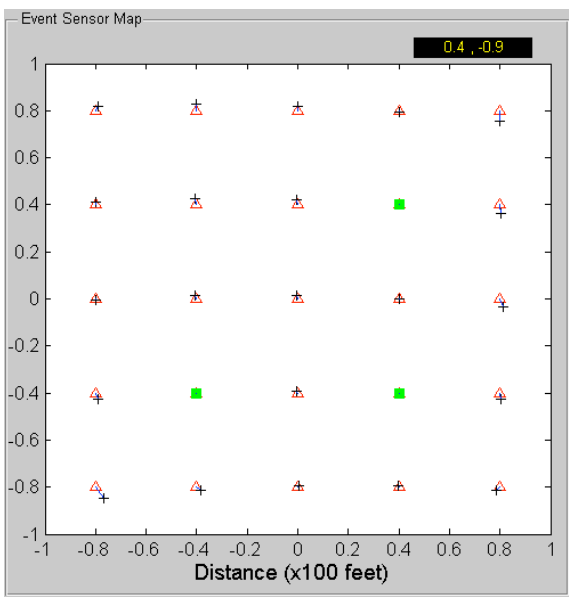

Fig. 5. Localization on a sparse network with anchor nodes deployed at the center of the sensor bed.

The localization error, $L_{e r r}$, improved to $5.75 \%$ when the anchor nodes were fixed around the center, as shown in Fig. 5. This may be justified due to the global events occurring at a large distance from the network. Due to this, the difference in projected distances would be approximately proportional to the physical separation between the nodes.

Next, the sensor network was scaled to accommodate 81 sensor nodes in a $9 \times 9$ grid. This time, 4 anchor nodes were used. We first placed these anchor nodes at the perimeter of the network to observe the localization. The localization error achieved is $18.63 \%$ in Fig. 6 , as compared to $12.24 \%$ when anchors were placed at the center in Fig. 7 . 


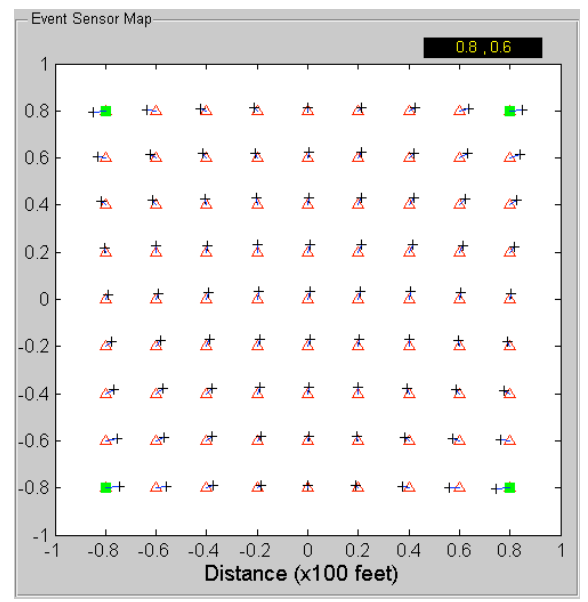

Fig. 6. Localization on a dense network with anchor nodes deployed at the perimeter of the sensor bed.

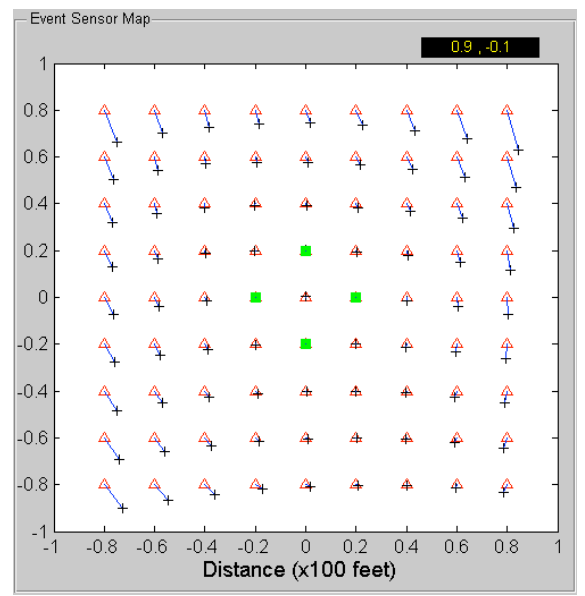

Fig. 7. Localization with anchor nodes deployed at the center of the sensor bed.

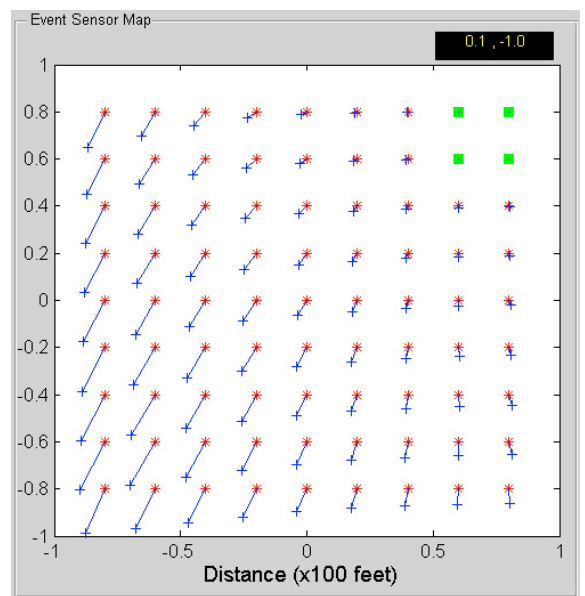

Fig. 8. Localization with anchor nodes deployed at the corner of the sensor bed.

A localization error, $L_{\text {err }}$, of $43.75 \%$ was realized in Fig. 8. The localization error became very erroneous when the anchors were placed at the corner. Our results show better localization is achieved when the anchors are placed at the center of the sensor network. Finally, we conducted a study on how the anchor nodes should be placed for effective localization.

One can see that anchors should never be linearly related. We placed 3 anchor nodes at the center of the network, which gave rise to erratic localization error of $586.54 \%$ in Fig. 9 .

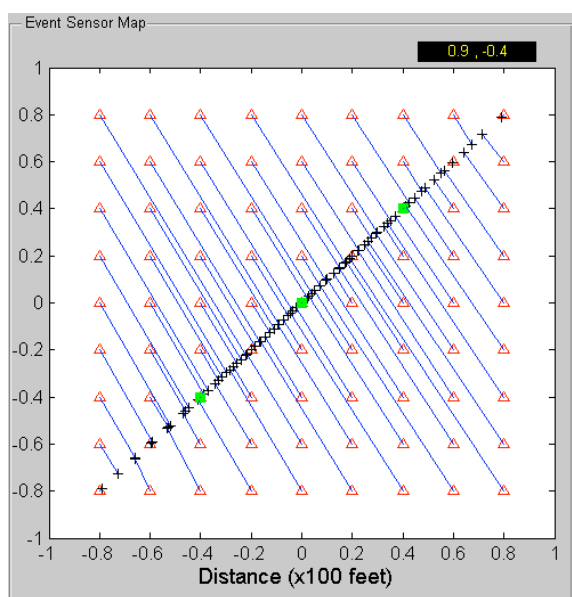

Fig. 9. Localization with anchor nodes deployed linearly around the center of the sensor bed.

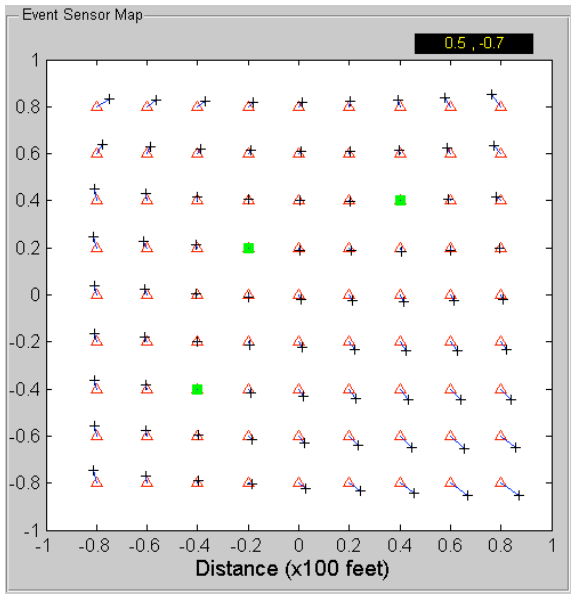

Fig. 10. Localization with anchor nodes deployed non-linearly around the center of the sensor bed.

On the other hand, when the angle between the anchor nodes was increased, hence increasing the non-linearity between them, it resulted in much better localization of $L_{e r r}=$ $14.92 \%$, as show in Fig. 10 .

Placing anchors at an acute angle as in Fig. 11 ended up with localization error, $L_{e r r}=14.11 \%$, similar to the previous one. A better localization error, $L_{e r r}$, of $11.57 \%$ was achieved when placing the anchor nodes to form a right angle, as in Fig. 12. 


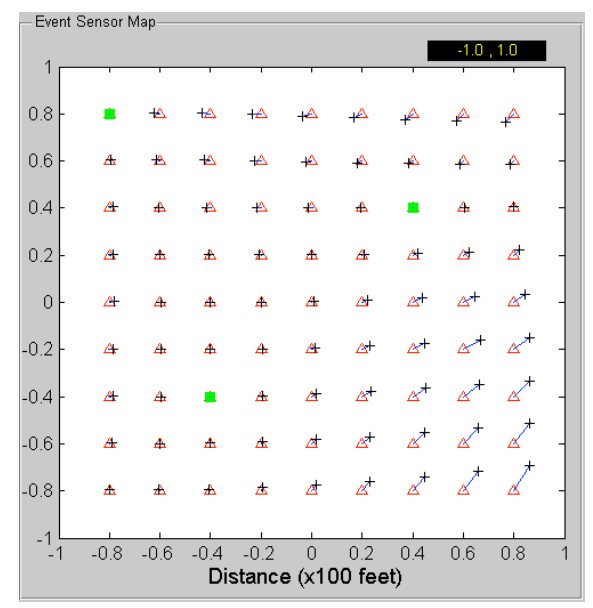

Fig. 11. Localization with anchor nodes deployed at non-linear positions.

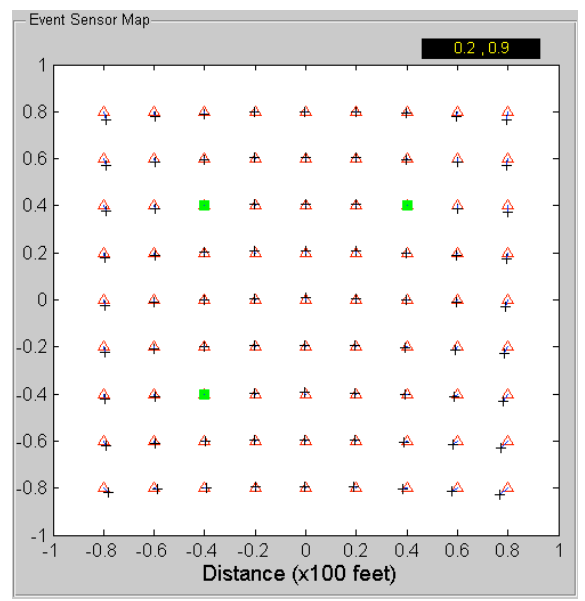

Fig. 12. Localization with anchor nodes deployed to form a right angle.

Finally, increasing the number of anchor nodes with a linear relationship was ineffective as depicted in Fig. 13, where $L_{\text {err }}=11.54 \%$.

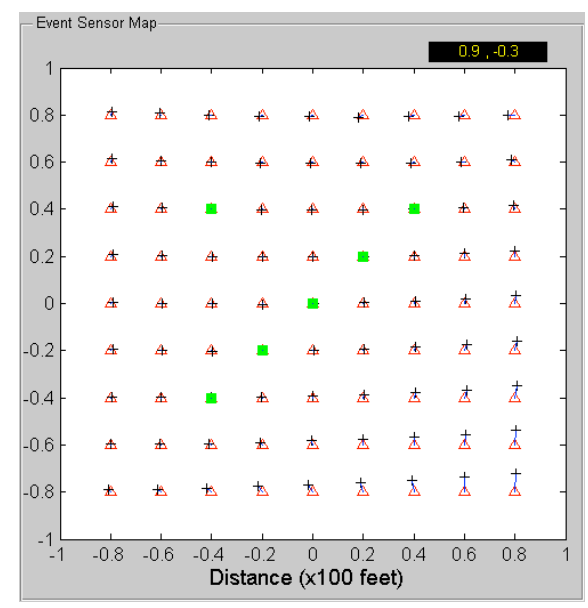

Fig. 13. Localization with extra anchor nodes deployed at linear positions.

\section{CONCLUSIONS}

In this paper, we analyzed the effects of the positions of anchor nodes on localization in sensor networks. Our simulation results show that, for effective passive localization, the optimal placement of the anchor nodes is at the center of the network in such a way that no three anchor nodes share linearity. The more the anchor nodes are non-linear, the smaller the localization error. Furthermore, the localization seemed to be best when the anchor nodes are placed to form a right angle. The positioning works on both sparse and dense networks.

\section{REFERENCES}

[1] J. Hill, R. Szewczyk, A. Woo, S. Hollar, D. Culler and K. Pister, "System Architecture Directions for Networked Sensors", ASPLOS, 2000.

[2] C. P. Townsend, S. W. Arms, "Wireless Sensor Networks: Principles and Applications", Sensor Technology Handbook, pp. 439-449, 2004.

[3] Y. Kwon and G. Agha, "Passive localization: Large size sensor network localization based on environmental events", International Conference on Information Processing in Sensor Networks, pp. 3-14, 2008.

[4] J. Bachrach, C. Taylor, "Localization in Sensor Networks", Handbook of Sensor Networks, pp. 277-310, 2005.

[5] J. N. Ash, R. L. Moses, "On optimal anchor node placement in sensor localization by optimization of subspace principal angles", Proceedings of IEEE International Conference on Acoustics, Speech, and Signal Processing, pp. 2289-2292, 2008.

[6] N. A. Alsindi, K. Pahlavan, "Node Localization", Wireless Sensor Networks: A Networking Perspective, pp. 243-284, 2009.

[7] A. Boukerche, H. A. B. F. Oliveira, E. F. Nakamura, and A. A. Loureiro, "Localization systems for wireless sensor networks", IEEE Wireless Communications, vol. 14, no.6, pp. 6-12, 2007.

[8] D. C. Lay, "Linear Algebra and Its Applications", Addison Wesley, 1994. 